\title{
Transition Mechanism of InAs Quantum Dot to Quantum Ring Revealed by Photoluminescence Spectra
}

\author{
Jong-Horng Dai, Jheng-Han Lee, and Si-Chen Lee, Fellow, IEEE
}

\begin{abstract}
The transition mechanism of InAs quantum dot (QD) to quantum ring $(\mathrm{QR})$ was investigated. After the growth of InAs QDs, a thin layer of GaAs was overgrown on the InAs QD and the sample was annealed at the same temperature for a period of time. It was found that the central part of the InAs islands started to out diffuse and formed ring shape only after a deposition of a critical thickness $(1 \sim 2 \mathrm{~nm})$ of GaAs capped layer depending on the size of InAs QDs. This phenomenon was revealed by photoluminescence measurement and atomic force microscopy image. It is suggested that the strain energy provided by the GaAs overgrown layer is responsible for the InAs to diffuse out of the island to form QR.
\end{abstract}

Index Terms-Blue-shifted, outward diffusion, quantum dot (QD), quantum ring (QR).

\section{INTRODUCTION}

$\mathbf{T}$ HE In(Ga)As quantum rings (QRs) are typically formed by first growing the InAs quantum dots (QDs) on a GaAs substrate followed by an immediate deposition of a thin GaAs capped layer, i.e., several nanometers, then annealing the sample at the growth temperature for a period of time. The formation mechanism has been attributed to the outward diffusion of In adtoms from the central part of InAs islands [1]-[3]. It was found that under properly prepared QD size and GaAs overgrown layer thickness [4]-[6], the top region of uncovered InAs QD tends to out diffuse and pile up around the center to a ring-shape after a few seconds of growth interruption. The diffusion out process is the migration of the In adatoms on the InAs island to a neighboring GaAs capped layer and intermixed with GaAs to form an In $(\mathrm{Ga})$ As alloy. The morphologies of QRs and QDs are quite different [7]-[9]. The realistic structure of the QR can be analyzed by atomic force microscopy (AFM), transmission electron microscopy, and scanning tunneling microcopy [10]-[12]. However, the process is difficult to monitor in situ during the growth, there is no elegant method to observe clearly the transformation of a dot into a ring, although the reflection high energy electron diffraction and intensity oscillations can in principle be used to monitor the transformation in situ, [9] but the detailed diffusion mechanism is still not clear. In this letter, we present

Manuscript received January 10, 2008; revised April 16, 2008. This work was supported by the National Science Council of the Republic of China under Contract NSC 96-2221-E-002-042.

The authors are with the Department of Electrical Engineering and Graduate Institute of Electronics Engineering, National Taiwan University, Taipei, 10617 Taiwan, R.O.C.

Color versions of one or more of the figures in this letter are available online at http://ieeexplore.ieee.org.

Digital Object Identifier 10.1109/LPT.2008.926813
TABLE I

DENSITY of InAs QDs CATEGORIZED BY QD HeIGHT; ALL QDs ARE CAPPED BY GaAs LAYER WITH VARIOUS THICKNESSES

\begin{tabular}{crrrrr}
$\begin{array}{c}\text { QD height } \\
(\mathrm{nm})\end{array}$ & \multicolumn{6}{c}{ GaAs thickness $(\mathrm{nm})$ capped on InAs QDs } \\
\cline { 2 - 6 } & 0 & 0.3 & 1 & 1.5 & 2 \\
\hline \hline$>17$ & 6 & 0 & 0 & 0 & 0 \\
\hline $12 \sim 17$ & 32 & 6 & 0 & 0 & 0 \\
\hline $7 \sim 12$ & 106 & 72 & 62 & 0 & 0 \\
\hline $3 \sim 7$ & 278 & 259 & 40 & 56 & 0 \\
\hline$<3$ & 201 & 218 & 10 & 41 & 0 \\
\hline \hline
\end{tabular}

Number of QDs (per square micrometer).

our results on the InAs diffusion out process by capping the InAs islands with a series of GaAs layers with different thicknesses. The morphology of all samples was measured and the QD/QR size and density were enumerated. Comparing the results from different samples, the out-diffusion mechanisms of InAs atoms and the QD size-dependent critical GaAs overgrown thickness were revealed clearly.

\section{EXPERIMENTS AND RESULTS}

The samples were grown by solid-source molecular beam epitaxy (MBE) on semi-insulating (100) GaAs substrates using a VG Semicon MBE machine. The original InAs QD sample without a GaAs capped layer is grown with 2.6 monolayer (ML) InAs coverage at $490^{\circ} \mathrm{C}$ at a growth rate of $\sim 0.1 \mathrm{ML} / \mathrm{s}$; then, the In cell shutter was closed and the InAs QD was annealed at the growth temperature for $\sim 30 \mathrm{~s}$. The samples were subsequently capped with 0.3-, 1-, 1.5-, and 2.0-nm GaAs layers, respectively, and annealed for $\sim 30 \mathrm{~s}$ at the same temperature. Finally, the substrate heater was turned OFF immediately after annealing. The QD height is measured from the surface of the GaAs capped layer to the top of the QD by AFM. The portion of QDs buried under the GaAs capped layer is not counted. The density of QDs categorized by QD heights are enumerated in a $1 \mu \mathrm{m} \times 1 \mu \mathrm{m}$ area and listed in Table I. It is clear that the distribution of QDs can be sorted into five groups according to their heights. From the AFM image in Fig. 1(a), it is observed that wide distribution of a small coherent island (with QD height less than $7 \mathrm{~nm}$ ) and a large dislocated island [(DI) with QD height larger than $7 \mathrm{~nm}$ ] with total density about $6 \times 10^{10} \mathrm{~cm}^{-2}$ are present in a 2.6-ML InAs QD sample without a GaAs capped layer [13]. The AFM images of InAs QDs with GaAs capped layer thickness from $0.3,1,1.5$, to $2 \mathrm{~nm}$ were displayed in Figs. 1(b), (c), (d), and (e), respectively. The growth mechanisms of each sample are illustrated in their right column. The InAs QD formed by the dewetting phenomenon was sketched in the right column of Fig. 1(a). 

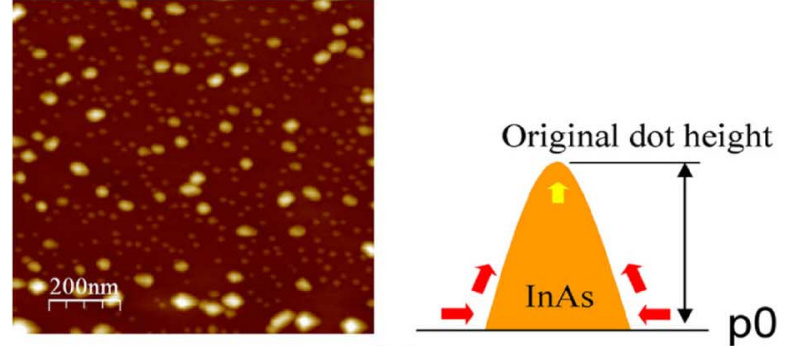

(a)
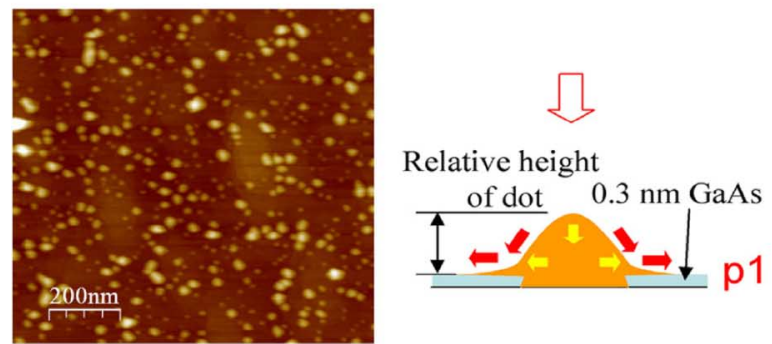

(b)
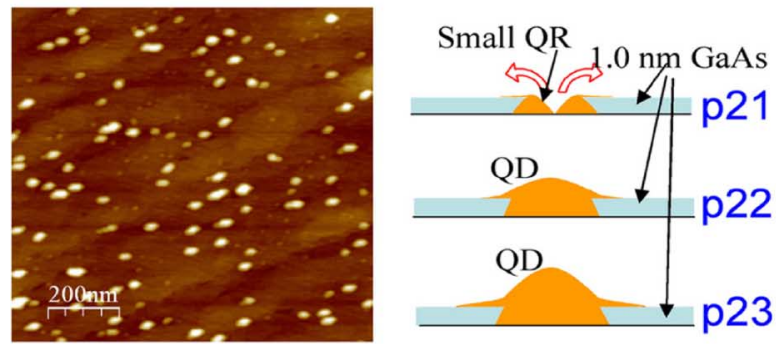

(c)
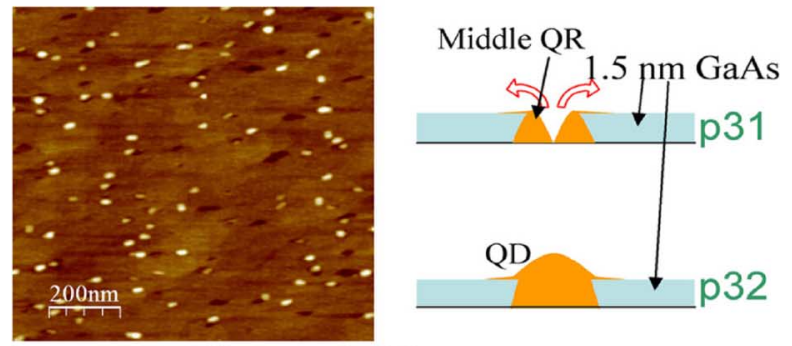

(d)
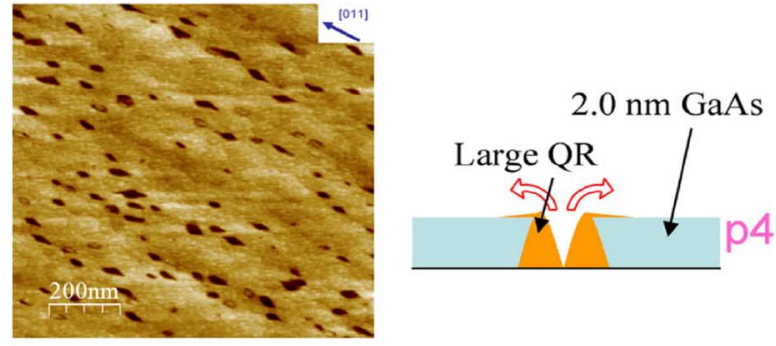

(e)

Fig. 1. AFM images of surface InAs QD capped with (a) 0-, (b) 0.3-, (c) 1-, (d) 1.5-, and (e) 2-nm GaAs layer and annealed for $30 \mathrm{~s}$. The growth mechanisms of each sample are illustrated in the right column.

The dewetting phenomenon explains the QD to QR transition that the center part of InAs QD which are buried in the GaAs is expelled to form the hole [4]-[6]. Comparing the original QD sample and that with the 0.3-nm GaAs capped layer [Fig. 1(b)], the average QD height was reduced, but its diameter increased

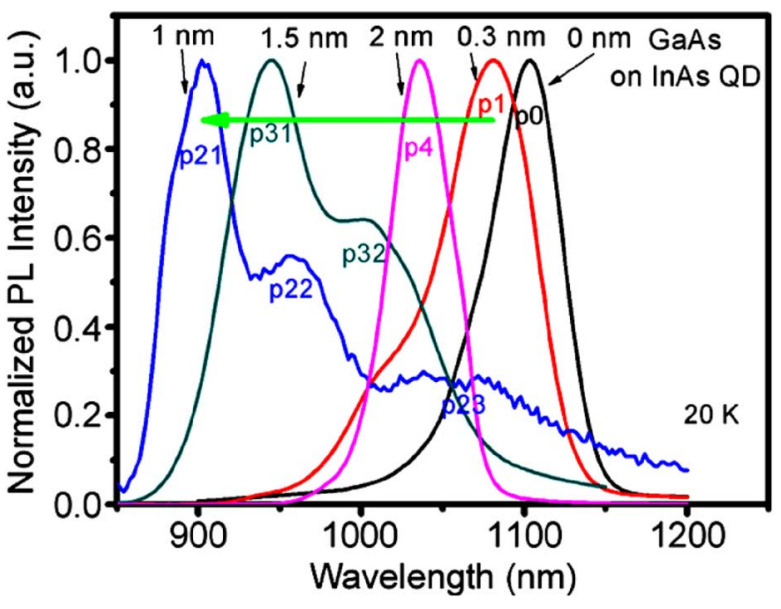

Fig. 2. PL spectra of InAs QDs capped with different GaAs layer thicknesses.

as illustrated in the right column of Fig. 1(b). The densities of large DIs become lower than the uncapped DIs, but the DIs are too high to let In adatoms to diffuse all out of the islands, so the InAs QDs still remain the typical shape. Those In adatoms migrated toward the GaAs surface will intermix with GaAs to form $\operatorname{In}(\mathrm{Ga})$ As alloy. The reduction of QD height is due to the burying of part of the InAs island and outward diffusion of In adatoms from the unburied InAs island. The large QDs lose their height with the increasing GaAs capped layer thickness until they totally disappear. The density of QDs decreases with increasing GaAs capped layer thickness as listed in Table I. When the GaAs capped layer thickness is lower than $1.5 \mathrm{~nm}$, it is difficult to find the QRs from AFM images [Fig. 1(b) and (c)] because the morphology does not change very much from that of the QD sample. The morphology of the 1.5-nm GaAs capped sample displayed in Fig. 1(d) shows both dots and rings. For the 2.0-nm GaAs capped sample, the islands all disappear and the hole-shape rings appear. It demonstrates that a smaller size QD will transform to QR at smaller GaAs capped layer thickness.

It is clear that for the 1.5- and 2.0-nm GaAs capped samples, the large QR holes and QD shape are both elongated along the [011] direction. This is consistent with the reports [9], [14], [15] that In adatom has a longer migration length in [011] direction on the GaAs surface, hence the large dots and rings appear to have a noncircular shape.

For photoluminescence (PL) measurement, the samples with the same structures as those described in Fig. 1(b)-(d) were further capped with another 100-nm GaAs layer to increase PL efficiency. Samples were excited using an $\mathrm{Ar}^{+}$laser at a 514.5-nm wavelength with a spot size of $300 \mu \mathrm{m}$. The PL spectrum was detected through a $0.5-\mathrm{m}$ triple grating monochromator using an InGaAs detector whose detection range was below $1.7 \mu \mathrm{m}$.

\section{DISCUSSION}

The $20 \mathrm{~K}$ PL spectra of those samples are shown in Fig. 2. The QD sample without the GaAs capped layer exhibits peak wavelength at $1104 \mathrm{~nm}(\mathrm{P} 0)$. After capping 0.3-nm GaAs layer and annealing, the In atoms at the exposed InAs QD region out-diffuse and are mixed with neighboring GaAs capped layer to form $\operatorname{In}(\mathrm{Ga})$ As with an equilibrium shape [2]. The QD height is reduced slightly, hence the peak wavelength is slightly blueshifted to $\sim 1080 \mathrm{~nm}(\mathrm{P} 1)$. When the QDs were capped with 
1-nm GaAs layer, the PL spectra exhibit dramatic changes indicating the emergence of new structure. Three peaks are observed; the dramatically blue-shifted one at $902 \mathrm{~nm}$ (P21) may be due to the formation of $\operatorname{In}(\mathrm{Ga}) \mathrm{As} \mathrm{QRs}$ which has been transformed from the small size InAs QDs. The ring height of the QRs are determined by the 1.0-nm GaAs capped layer and thus are very uniform. These uniform QRs give rise to strong PL peak. Many of the medium sized QDs lost their heights which result in a broader middle peak at $958 \mathrm{~nm}(\mathrm{P} 22)$, the rest of larger QDs with the height from 7 to $12 \mathrm{~nm}$ give rise to the broadest peak at $1054 \mathrm{~nm}$ (p23) as illustrated in the right column of Fig. 1(c). As for the 1.5-nm GaAs capped QDs, the dominant highest energy peak red shifts to $946 \mathrm{~nm}$ (P31) which is due to the higher QR height fixed by the 1.5-nm capped GaAs layer thickness and transformed from small and medium sized QDs. The originally large QDs which remained in the dot-shape exhibit a smaller peak at $1000 \mathrm{~nm}$ (P32) as sketched in the right column of Fig. 1(d). Finally, for the 2-nm GaAs capped sample, all of the QDs were transformed to QRs. The PL spectrum shows a single peak at $1036 \mathrm{~nm}(\mathrm{P} 4)$. Since no QDs exists, all QRs have the same height $(2.0 \mathrm{~nm})$ fixed by the GaAs capped layer thickness, and the PL spectra exhibit narrow and strong peak. It clearly demonstrates that the $\mathrm{QR}$ formation mechanism is due to the outward diffusion of In atoms from the uncovered InAs QDs. The GaAs capped layer thickness determines the height and optical property of QRs.

It is observed that the 2-nm GaAs capped layer enables the InAs in the central part of the QDs to diffuse all the way out of the dots to form QRs whether the original dot height is $<3$ or $>17 \mathrm{~nm}$. The 1.5-nm GaAs capped layer is unable to achieve this effect. Many large sized QDs still remain the typical shape without transforming totally into QRs. Only their heights are reduced. Although that is only a $0.5-\mathrm{nm}$ difference between the two capped GaAs layer thicknesses, the morphologies of two samples are significantly different. This demonstrates that the thicker GaAs capped layer, i.e., $2 \mathrm{~nm}$, provides enough strain energy to force InAs diffusing out of the large sized InAs QD and form QR. The outward diffusion phenomenon is driven by strain energy caused by overgrown GaAs layer.

\section{CONCLUSION}

This study fully supports the formation mechanism of QRs to outward diffusion of central QDs. A critical GaAs capped layer thickness of $1 \mathrm{~nm}$ is necessary for the transformation of smaller InAs QDs to QRs, whereas a 2-nm GaAs capped layer is required for larger QDs. The outward diffusion phenomenon is driven by strain energy caused by an overgrown GaAs layer. Since the QR height is determined by the GaAs capped layer thickness, they become very uniform and give rise to strong and narrow PL peaks. This suggests that the optical properties of the
QRs are tailorable with the GaAs capped layer thickness. Therefore, those characteristics of $\mathrm{QR}$ are very useful for application in optoelectronic devices in the terahertz region.

\section{ACKNOWLEDGMENT}

The authors would like to thank Prof. H. H. Lin of the Department of Electrical Engineering, National Taiwan University for helpful discussions of PL measurement.

\section{REFERENCES}

[1] A. Lorke, R. Blossey, J. M. Garcia, M. Bichler, and G. Abstreiter, "Morphological transformation of $\operatorname{In}_{y} \mathrm{Ga}_{1-y}$ As islands, fabricated by Stranski-Krastanov growth," Mater. Sci. Eng., vol. B88, pp. 225-229, 2002.

[2] A. Lorke, R. J. Luyken, J. M. Garcia, and P. M. Petroff, "Growth and electronic properties of self-organized quantum rings," Jpn. J. Appl. Phys., vol. 40, pp. 1857-1859, 2001.

[3] M. Sztucki, T. H. Metzger, V. Chamard, A. Hesse, and V. Holy, "Investigation of shape, strain, and interdiffusion in InGaAs quantum rings using grazing incidence X-ray diffraction," J. Appl. Phys., vol. 99, pp. $0335191-0335199,2006$.

[4] K. Takehana, F. Pulizzi, A. Patane, M. Henini, P. C. Main, L. Eaves, D. Granados, and J. M. Garcia, "Controlling the shape of InAs self-assembled quantum dots by thin GaAs capping layers," J. Cryst. Growth, vol. 251, pp. 155-160, 2003.

[5] D. Granados and J. M. Garcia, "In(Ga)As self-assembled quantum ring formation by molecular beam epitaxy," Appl. Phys. Lett., vol. 82, pp. 2401-2403, 2003.

[6] J. H. Dai, J. H. Lee, and S. C. Lee, "Annealing effect on the formation of $\operatorname{In}(\mathrm{Ga})$ As quantum rings from InAs quantum dots," IEEE Photon. Technol. Lett., vol. 20, no. 2, pp. 165-167, Jan. 15, 2008.

[7] A. Lorke, R. J. Luyken, A. O. Govorov, J. P. Kotthaus, J. M. Garcia, and P. M. Petroff, "Spectroscopy of nanoscopic semiconductor rings," Phys. Rev. Lett., vol. 84, pp. 2223-2226, 2000.

[8] J. I. Climente, J. Planelles, and W. Jaskolski, "Magneto-optical transitions in nanoscopic rings," Phys. Rev. B, vol. 68, pp. 075307 1-075307 8, 2003.

[9] J. M. Garcia, D. Grandos, J. P. Silveira, and F. Briones, "In segregation effects during quantum dot and quantum ring formation on GaAs(001)," Microelectron. J., vol. 35, pp. 7-11, 2004.

[10] P. Offermans, P. M. Koenraad, J. H. Wolter, D. Granados, J. M. Garcia, V. M. Fomin, V. N. Gladilin, and J. T. Devreese, "Atomic-scale structure and formation of self-assembled $\operatorname{In}(\mathrm{Ga})$ As quantum rings," Phys. E, vol. 32, pp. 41-45, 2006.

[11] D. Granados, J. M. Garcia, T. Ben, and S. I. Molina, "Vertical order in stacked layers of self-assembled $\mathrm{In}(\mathrm{Ga})$ As quantum rings on GaAs (001)," Appl. Phys. Lett., vol. 86, pp. 071918 1-071918 3, 2005.

[12] S. Suraprapapich, S. Panyakeow, and C. W. Tu, "Effect of arsenic species on the formation of (Ga)InAs nanostructures after partial capping and regrowth," Appl. Phys. Lett., vol. 90, pp. 183112 1-183112 3, 2007.

[13] S. Guha, A. Madhukar, and K. C. Rajkumar, "Onset of incoherency and defect introduction in the initial stages of molecular beam epitaxical growth of highly strained $\mathrm{In}_{x} \mathrm{Ga}_{1-x}$ As on GaAs(100)," Appl. Phys. Lett., vol. 57, pp. 2110-2112, 1990.

[14] J.-S. Lee, H.-W. Ren, S. Sugou, and Y. Masumoto, “ $\mathrm{In}_{0.5} \mathrm{Ga}_{0.5} \mathrm{As}$ quantum dot intermixing and evaporation in GaAs capping layer growth," J. Appl. Phys., vol. 84, pp. 6686-6688, 1998.

[15] J. M. Garcia, G. M. Ribeiro, K. Schmidt, T. Ngo, J. L. Feng, A. Lorke, J. Kotthaus, and P. M. Petroff, "Intermixing and shape changes during the formation of InAs self-assembled quantum dots," Appl. Phys. Lett., vol. 71, pp. 2014-2016, 1997. 